\title{
Synthesis and Hydrogenation of Bis(imino)pyridine Iron Imides
}

\author{
Suzanne C. Bart, Emil Lobkovsky, Eckhard Bill' and Paul J. Chirik** \\ pc92@cornell.edu \\ Department of Chemistry and Chemical Biology, Baker Laboratory, Cornell University, \\ Ithaca NY 14853. \\ ${ }^{\dagger}$ Max-Plank Institute of Bioinorganic Chemistry, Shiftstrasse 34-36, D-45470 Mülheim an \\ der Ruhr, Germany \\ -- REVISED -- \\ Supporting Information
}




\section{Table of Contents}

$\begin{array}{ll}\text { Experimental Details } & \text { S3 }\end{array}$

$\begin{array}{ll}\text { Procedure for the Deuteration of }\left(\mathbf{1}-\mathbf{N}_{2}\right)_{2} \text { and } \mathbf{2 a} & \text { S7 }\end{array}$

$\begin{array}{ll}\text { Details of Catalytic Azide Hydrogenation } & \text { S8 }\end{array}$

$\begin{array}{ll}\text { Crystallographic data for } \mathbf{2 a} & \text { S9 }\end{array}$

$\begin{array}{lr}\text { Crystallographic data for } \mathbf{2 d} & \text { S14 }\end{array}$

$\begin{array}{lr}\text { Comparison the solid state structure of } \mathbf{2 a} \text { and } \mathbf{2 d} & \text { S18 }\end{array}$

$\begin{array}{ll}\text { Qualtitative correlation diagram for bis(imino)pyridine iron imides } & \text { S19 }\end{array}$

$\begin{array}{lr}{ }^{1} \mathrm{H} \text { NMR Spectrum of } \mathbf{2 d} & \text { S20 }\end{array}$

$\begin{array}{ll}\text { Cyclic Voltammogram of 2a } & \text { S21 }\end{array}$

$\begin{array}{ll}\text { References } & \text { S22 }\end{array}$ 


\section{Experimental Section}

General Considerations. All air- and moisture-sensitive manipulations were carried out using standard vacuum line, Schlenk and cannula techniques or in an MBraun inert atmosphere drybox containing an atmosphere of purified nitrogen. The MBraun drybox was equipped with a cold well designed for freezing samples in liquid nitrogen. Solvents for air- and moisture-sensitive manipulations were initially dried and deoxygenated using literature procedures. ${ }^{1}$ Argon and hydrogen gas were purchased from Airgas Incorporated and passed through a column containing manganese oxide supported on vermiculite and 4 $\AA$ molecular sieves before admission to the high vacuum line. Benzene- $d_{6}$ was purchased from Cambridge Isotope Laboratories and distilled from sodium metal under an atmosphere of argon and stored over $4 \AA$ molecular sieves or sodium metal. Both the aryl azides $^{2}$ and 1-( $\left.\mathbf{N}_{2}\right)_{2}{ }^{3}$ were prepared according to literature procedures.

${ }^{1} \mathrm{H}$ NMR spectra were recorded on Varian Mercury 300, Inova 400 and 500 spectrometers operating at 299.763, 399.780 and $500.62 \mathrm{MHz}$, respectively. All chemical shifts are reported relative to $\mathrm{SiMe}_{4}$ using ${ }^{1} \mathrm{H}$ (residual) chemical shifts of the solvent as a secondary standard. For paramagnetic molecules, the ${ }^{1} \mathrm{H}$ NMR data are reported with the chemical shift followed by the peak width at half height in Hertz followed by integration value and where possible, peak assignment.

Single crystals suitable for X-ray diffraction were coated with polyisobutylene oil in a drybox and were quickly transferred to the goniometer head of a Siemens SMART CCD Area detector system equipped with a molybdenum X-ray tube $(\lambda=0.71073 \AA$ ). Preliminary data revealed the crystal system. A hemisphere routine was used for data collection and determination of lattice constants. The space group was identified and the 
data were processed using the Bruker SAINT program and corrected for absorption using SADABS. The structures were solved using direct methods (SHELXS) completed by subsequent Fourier synthesis and refined by full-matrix least-squares procedures.

Cyclic voltammograms (CVs) were collected using $30 \mathrm{~mL}$ beakers as electrochemical cells with a $3 \mathrm{~mm}$ glassy carbon working electrode, Pt wire as a counter electrode, and $\mathrm{Ag}$ wire as a reference in a drybox equipped with electrochemical outlets. CVs were recorded using a Bioanalytical Systems CV-27 voltammograph. All CVs were run at a scan rate of $100 \mathrm{mV} / \mathrm{s}$. Solutions of the individual compounds were prepared by charging $4 \mathrm{mg}$ of $\mathbf{2 a}$ and $0.400 \mathrm{~g}(1.03 \mathrm{mmol})$ of $\left[n-\mathrm{Bu}_{4} \mathrm{~N}\right]\left[\mathrm{PF}_{6}\right]$ in a vial and dissolving the solids in $10 \mathrm{~mL}$ of THF. This produced solutions of approximately $0.5 \mathrm{mM}$ in $\mathbf{2 a}$ and $0.1 \mathrm{M}$ in electrolyte. After recording the baseline of a standard $0.1 \mathrm{M}$ solution of electrolyte, CVs were collected for $\mathbf{2 a}$, including $\mathrm{Cp}_{2} \mathrm{Fe}$. Redox potentials were then referenced to the formal potential of ferrocene.

Mössbauer data were collected on an alternating constant-acceleration spectrometer. The minimum experimental line width was $0.24 \mathrm{~mm} \mathrm{~s}^{-1}$ (full width at half height). A constant sample temperature was maintained with an Oxford Instruments Variox or an Oxford Instruments Mössbauer-Spectromag 2000 cyrostat. Reported isomer shifts ( $\delta)$ are referenced to iron metal at $293 \mathrm{~K}$.

Preparation of $\left[\left({ }^{i \mathrm{Pr}} \mathbf{P D I}\right) \mathrm{FeN}\left(\mathbf{2 , 6}-{ }^{\mathrm{i}} \mathbf{P r}_{2}-\mathbf{C}_{6} \mathbf{H}_{3}\right)\right]$ (2a). A $20 \mathrm{~mL}$ scintillation vial was charged with $0.060 \mathrm{~g}(0.10 \mathrm{mmol})$ of $\mathbf{1}-\left(\mathbf{N}_{2}\right)_{2}$ and approximately $10 \mathrm{~mL}$ of pentane forming a green solution. Via microsyringe, $20 \mu \mathrm{L}(\sim 0.101 \mathrm{mmol})$ of 2,6diisopropylphenylazide was added to the vial with stirring. Immediately upon addition, 
bubbling ensued followed by a color change to deep purple. After stirring for five minutes, the volatiles were removed in vacuo yielding $0.070 \mathrm{~g}(93 \%)$ of a dark purple solid identified as 2a. Analysis for $\mathrm{C}_{45} \mathrm{H}_{60} \mathrm{~N}_{4} \mathrm{Fe}$ : Calc. C, 75.82; H, 8.48; N, 7.86. Found C, 75.49; H, 8.61; N, 8.07. Magnetic susceptibility (benzene- $\left.d_{6}\right) \mu_{\text {eff }}=2.8 \mu_{\mathrm{B}} .{ }^{1} \mathrm{H}$ NMR (benzene- $\left.d_{6}\right): \delta=-68.1(67.7),-25.1(553),-0.93\left(565,12 \mathrm{H},{ }^{\mathrm{i}} \operatorname{Pr} M e\right),-0.50$ (19.4), -0.30 (550, 12H, ${ }^{\mathrm{P}} \mathrm{PrMe}$ ), 0.29 (51.3), 6.1 (200), 7.8 (34.1), 111.2 (698), 121.8 (1920), 131.0 (108). ${ }^{1} \mathrm{H}$ NMR $\left(-80{ }^{\circ} \mathrm{C}\right.$, toluene- $\left.d_{8}\right): \delta=-23.26(622.22),-13.67$ (673.55), -13.05 (614.51), -8.43 (201.53), -3.53 (75.35), -1.45 (345.49), 10.65 (830.40), 11.54 (772.81), 18.84 (433.87).

Preparation of $\left[\left({ }^{\mathrm{iPr}} \mathbf{P D I}\right) \mathrm{FeN}\left(\mathbf{2 , 6}-\mathbf{E t}_{2}-\mathrm{C}_{6} \mathbf{H}_{3}\right)\right](\mathbf{2 b})$. This compound was prepared in an analogous manner to $\mathbf{2 a}$ with $0.050 \mathrm{~g}(0.084 \mathrm{mmol})$ of $\mathbf{1}-\left(\mathbf{N}_{2}\right)_{2}$ and $15 \mu \mathrm{L}(\sim 0.084 \mathrm{mmol})$ of 2,6-diethylphenyl azide yielding $0.041 \mathrm{~g}$ (72\%) of a dark blue-purple solid identified as 2b. Analysis for $\mathrm{C}_{43} \mathrm{H}_{56} \mathrm{~N}_{4} \mathrm{Fe}$ : Calc. C, 75.42; H, 8.24; N, 8.18. Found $\mathrm{C}, 75.25 ; \mathrm{H}$, 8.62; N, 7.89. Magnetic susceptibility (benzene- $d_{6}$ ) $\mu_{\text {eff }}=2.8 \mu_{\mathrm{B}} .{ }^{1} \mathrm{H}$ NMR (benzene- $d_{6}$ ): $\delta$ $=-90.1(87.9,6 \mathrm{H}, \mathrm{N}=\mathrm{C}(M e)),-31.0(243.8,4 \mathrm{H}),-3.7\left(60.7,12 \mathrm{H},{ }^{\mathrm{i}} \operatorname{Pr} M e\right),-3.4(37.5,12 \mathrm{H}$, $\left.{ }^{i} \operatorname{Pr} \mathrm{Me}\right), 0.01(31.4,4 \mathrm{H}), 0.58\left(59.3,6 \mathrm{H}, \mathrm{CH}_{2} \mathrm{CH}_{3}\right), 3.3\left(29.4,4 \mathrm{H}, \mathrm{CH}_{2} \mathrm{CH}_{3}\right), 24.1$ (48.6, 2H), 127.51 (98.91, 1H, p-pyr), 134.6 (209.1, 2H), 272.1 (489.3, 2H).

Preparation of $\left[\left({ }^{\mathrm{iPr}} \mathrm{PDI}\right) \mathrm{FeN}\left(\mathbf{2 , 5}-{ }^{\mathrm{t}} \mathrm{Bu}_{2}-\mathrm{C}_{6} \mathrm{H}_{3}\right)\right](\mathbf{2 c})$. This compound was prepared in an analogous manner to $\mathbf{2 a}$ with $0.050 \mathrm{~g}(0.084 \mathrm{mmol})$ of $\mathbf{1 -}\left(\mathbf{N}_{2}\right)_{2}$ and $(\sim 0.084 \mathrm{mmol})$ of 2,5bis('butyl)phenyl azide yielding $0.059 \mathrm{~g}(95 \%)$ of a bright blue solid identified as $\mathbf{2 c}$. Analysis for $\mathrm{C}_{47} \mathrm{H}_{64} \mathrm{~N}_{4} \mathrm{Fe}$ : Calc. C, 76.19; H, 8.71; N, 7.56. Found C, 75.91; H, 8.62; N, 
7.47. Magnetic susceptibility (benzene- $d_{6}$ ) $\mu_{\mathrm{eff}}=3.0 \mu_{\mathrm{B}} \cdot{ }^{1} \mathrm{H}$ NMR (benzene- $d_{6}$ ): $\delta=-82.7$ (124.5, 6H, N=C(Me)), -44.3 (174.9, 2H), -30.3 (147.9, 2H), -19.7 (60.1, 6H, $\left.{ }^{\mathrm{i}} \operatorname{Pr} M e\right),-$ $11.3\left(22.6,6 \mathrm{H},{ }^{\mathrm{i}} \operatorname{Pr} M e\right),-4.3(21.6), 0.3\left(10.0,9 \mathrm{H},{ }^{t} \mathrm{Bu}\right), 0.5\left(27.2,6 \mathrm{H},{ }^{\mathrm{i}} \operatorname{Pr} M e\right), 3.3$ (26.8, 4H), $4.4\left(44.8,6 \mathrm{H},{ }^{\mathrm{i}} \mathrm{PrMe}\right), 5.6(21.9,2 \mathrm{H}), 18.3\left(130.25,9 \mathrm{H},{ }^{t} \mathrm{Bu}\right), 37.1(51.3,2 \mathrm{H}), 112.1$ (73.6, 1H), $131.3(144.1,1 \mathrm{H}), 214.5(162.9)$.

Preparation of $\left[\left({ }^{\mathrm{iPr}} \mathrm{PDI}\right) \mathrm{FeN}\left(\mathbf{2 , 4 , 6}-\mathrm{Me}_{3}-\mathrm{C}_{6} \mathrm{H}_{2}\right)\right](\mathbf{2 d})$. This compound was prepared in an analogous manner to $\mathbf{2 a}$ with $0.040 \mathrm{~g}(0.067 \mathrm{mmol})$ of $\mathbf{1}-\left(\mathbf{N}_{2}\right)_{2}$ and $10 \mu \mathrm{L}(\sim 0.067 \mathrm{mmol})$ of 2, 4, 6-trimethylphenylazide yielding $0.041 \mathrm{~g}(91 \%)$ of a bright blue solid identified as 2d. Analysis for $\mathrm{C}_{42} \mathrm{H}_{54} \mathrm{~N}_{4} \mathrm{Fe}$ : Calc. C, 75.21; H, 8.11; N, 8.35. Found C, 75.05; H, 8.49; $\mathrm{N}, 8.00$. Magnetic susceptibility (benzene- $d_{6}$ ) $\mu_{\mathrm{eff}}=2.8 \mu_{\mathrm{B}} .{ }^{1} \mathrm{H}$ NMR (benzene- $d_{6}$ ): $\delta=-$ $102.8(51.4,6 \mathrm{H}, \mathrm{N}=\mathrm{C}(M e)),-35.6(174.6,4 \mathrm{H}),-5.0\left(25.6,12 \mathrm{H},{ }^{\mathrm{i}} \operatorname{Pr} M e\right),-4.7(12.4,12 \mathrm{H}$, $\left.{ }^{\mathrm{i}} \operatorname{PrMe}\right),-0.3$ (15.1, 4H), 3.5 (15.8, 2H), 33.6 (25.5, 2H), 123.8 (47.5, 1H, p-pyr), 140.67 (114.2, 2H), $271.2(321.9,2 \mathrm{H})$.

General Procedure for Catalytic Azide Hydrogenation. A thick walled glass vessel was charged with $0.015 \mathrm{~g}(0.025 \mathrm{mmol})$ of $\mathbf{1 -}\left(\mathbf{N}_{2}\right)_{2}$ and approximately $3 \mathrm{~mL}$ of benzene. The resulting solution was frozen in the drybox coldwell and the azide (10 equivalents, $0.25 \mathrm{mmol}$ ) was added by microsyringe. The vessel was removed from the glovebox, immediately frozen in liquid nitrogen and evacuated. One atmosphere of hydrogen was added at $-196{ }^{\circ} \mathrm{C}$ and the vessel sealed with a Teflon stopcock. The contents of the vessel were thawed and the resulting reaction mixture stirred for 6 hours. The progress of the reaction was monitored by ${ }^{1} \mathrm{H}$ NMR spectroscopy. 


\section{Procedure for the Deuteration of 1-( $\left.\mathbf{N}_{2}\right)_{2}$ and 2a-2d.}

The isopropyl methyl groups of the aryl substituents in $\mathbf{1 -}\left(\mathbf{N}_{\mathbf{2}}\right)_{\mathbf{2}}$ were deuterated by exposure of the compound to one atmosphere of $\mathrm{D}_{2}$ for two days, as described

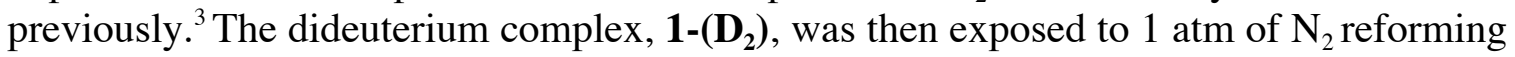
$\mathbf{1 -}\left(\mathbf{N}_{2}\right)_{2}$ which was subsequently treated with the appropriate aryl azide to yield $\mathbf{2 a - 2 d}$. A typical procedure is outlined below:

Procedure for Preparation of Deuterium Incorporated 1-( $\left.\mathbf{N}_{2}\right)_{2}$. A thick walled glass vessel was charged with $0.200 \mathrm{~g}(0.337 \mathrm{mmol}) \mathbf{1}-\left(\mathbf{N}_{2}\right)_{2}$ and $10 \mathrm{~mL}$ of pentane, forming a green solution. On the high vacuum line, the vessel was frozen in liquid nitrogen and evacuated. One atmosphere of deuterium gas was admitted at $-196{ }^{\circ} \mathrm{C}$ and the contents of the vessel warmed to ambient temperature. After stirring for two days, the volatiles were removed in vacuo to yield a dark green solid. This material was recrystallized from an ether:pentane mixture to yield $0.165 \mathrm{~g}(0.278 \mathrm{mmol}, 83 \%) \mathbf{1 -}\left(\mathbf{N}_{2}\right)_{2} \cdot{ }^{2} \mathrm{H}$ NMR spectroscopy established deuterium incorporation into the methyl groups of the isopropyl substituents of the aryl rings.

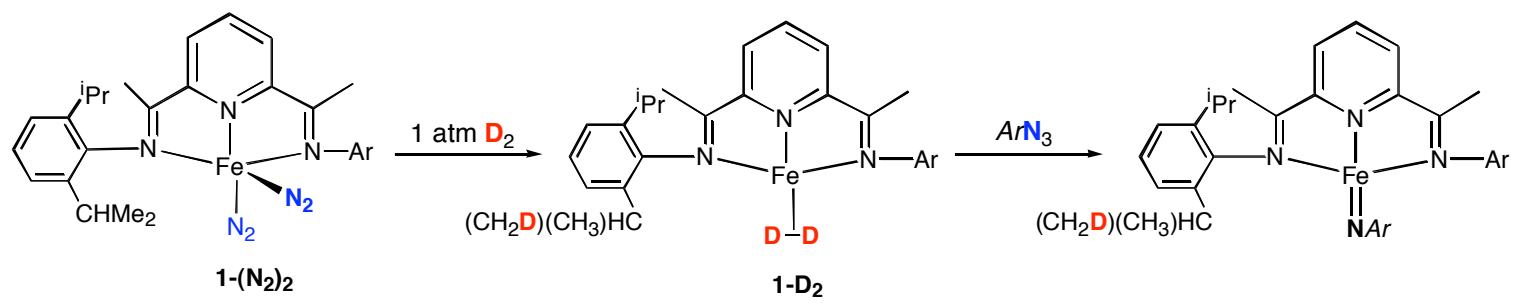




\section{Details of Catalytic Azide Hydrogenation}

\begin{tabular}{ccc}
\hline Compound & TOF $(\mathbf{m o l} / \mathbf{h r})$ & Temperature $\left({ }^{\mathbf{C}} \mathbf{C}\right)$ \\
\hline $\mathbf{2 a}$ & 1.7 & 23 \\
$\mathbf{2 b}$ & 0.10 & 65 \\
$\mathbf{2 c}$ & 0.60 & 65 \\
\hline
\end{tabular}




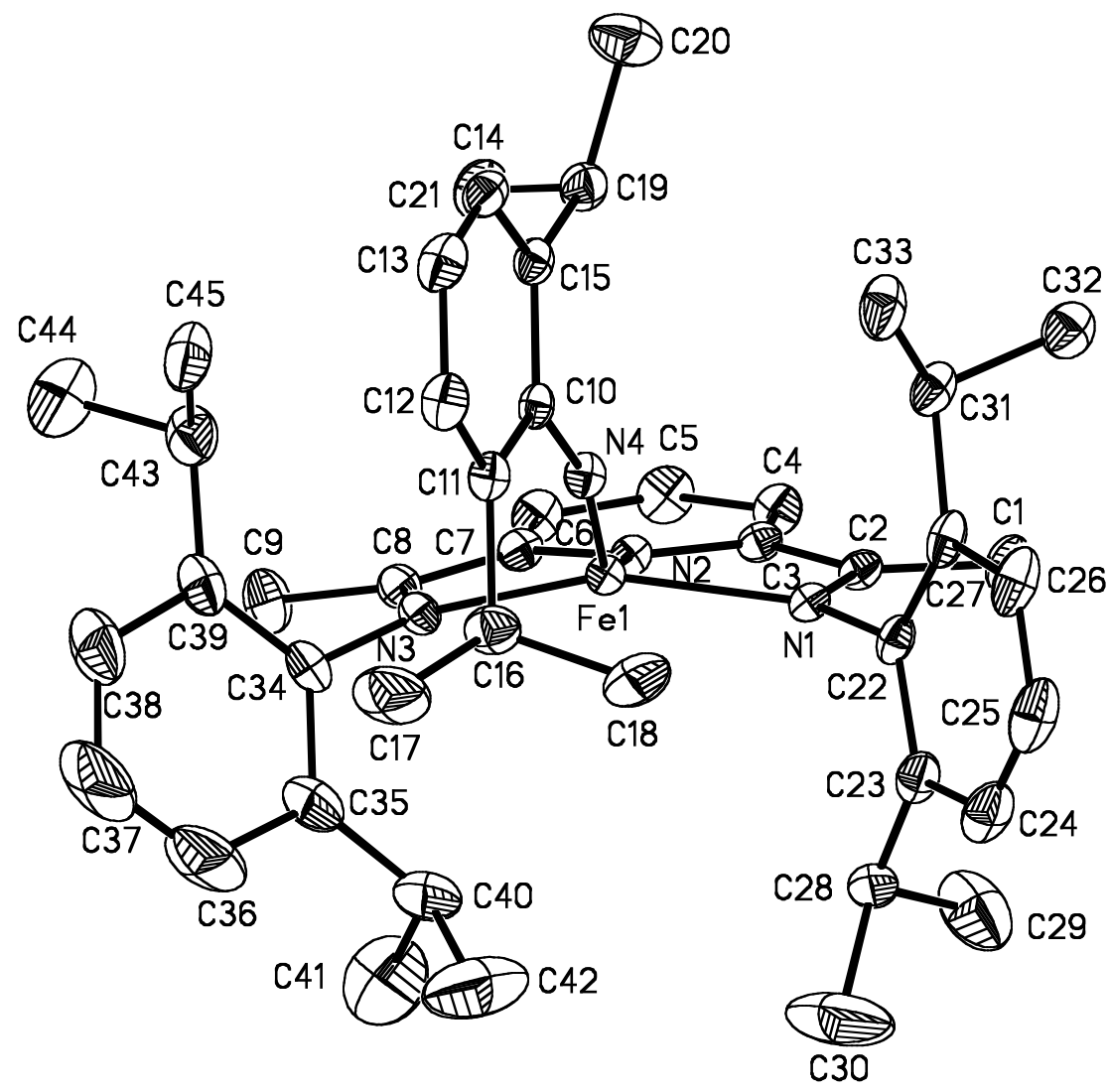

Figure S1. Fully labeled view of the molecular structure of $\mathbf{2 a}$ at $30 \%$ probability ellipsoids. Hydrogen atoms omitted for clarity. 
Table S1. Crystal data and structure refinement for $\mathbf{2 a}$

Identification code

Empirical formula

Formula weight

Temperature

Wavelength

Crystal system

Space group

Unit cell dimensions

Volume

Z

Density (calculated)

Absorption coefficient

$\mathrm{F}(000)$

Crystal size

Theta range for data collection

Index ranges

Reflections collected

Independent reflections

Completeness to theta $=28.28^{\circ}$

Absorption correction

Max. and min. transmission

Refinement method

Data / restraints / parameters

Goodness-of-fit on $\mathrm{F}^{2}$

Final $\mathrm{R}$ indices [I $>2 \operatorname{sigma}(\mathrm{I})]$

$\mathrm{R}$ indices (all data)

Largest diff. peak and hole scb44

C45 H60 Fe N4

712.82

173(2) K

$0.71073 \AA$

Monoclinic

$\mathrm{P} 2(1) / \mathrm{c}$

$\mathrm{a}=18.3869(6) \AA$

$\alpha=90^{\circ}$.

$\mathrm{b}=12.6293(4) \AA$

$\beta=103.199(1)^{\circ}$.

$c=17.7726(6) \AA$

$\gamma=90^{\circ}$.

4

$1.178 \mathrm{Mg} / \mathrm{m}^{3}$

$0.410 \mathrm{~mm}^{-1}$

1536

$0.35 \times 0.25 \times 0.15 \mathrm{~mm}^{3}$

1.97 to $28.28^{\circ}$.

$-24<=\mathrm{h}<=24,-9<=\mathrm{k}<=16,-23<=1<=23$

29143

$9509[\mathrm{R}($ int $)=0.0338]$

$95.4 \%$

Semi-empirical from equivalents

0.9410 and 0.8697

Full-matrix least-squares on $\mathrm{F}^{2}$

9509 / 0 / 511

1.058

$\mathrm{R} 1=0.0452, \mathrm{wR} 2=0.1156$

$\mathrm{R} 1=0.0750, \mathrm{wR} 2=0.1297$

0.574 and -0.393 e. $\AA^{-3}$ 
Table S2. Bond lengths $[\AA]$ and angles $\left[{ }^{\circ}\right]$ for $\mathbf{2 a}$

\begin{tabular}{|c|c|c|c|}
\hline $\mathrm{Fe}(1)-\mathrm{N}(4)$ & $1.7048(16)$ & $C(23)-C(28)$ & $1.524(4)$ \\
\hline $\mathrm{Fe}(1)-\mathrm{N}(2)$ & $1.8403(15)$ & $C(24)-C(25)$ & $1.376(4)$ \\
\hline $\mathrm{Fe}(1)-\mathrm{N}(3)$ & $1.9870(17)$ & $C(25)-C(26)$ & $1.363(4)$ \\
\hline $\mathrm{Fe}(1)-\mathrm{N}(1)$ & $2.0343(16)$ & $C(26)-C(27)$ & $1.394(3)$ \\
\hline $\mathrm{N}(1)-\mathrm{C}(2)$ & $1.320(2)$ & $\mathrm{C}(27)-\mathrm{C}(31)$ & $1.520(3)$ \\
\hline $\mathrm{N}(1)-\mathrm{C}(22)$ & $1.447(2)$ & $C(28)-C(29)$ & $1.468(4)$ \\
\hline $\mathrm{N}(2)-\mathrm{C}(7)$ & $1.373(3)$ & $C(28)-C(30)$ & $1.510(4)$ \\
\hline $\mathrm{N}(2)-\mathrm{C}(3)$ & $1.377(3)$ & $C(31)-C(33)$ & $1.530(3)$ \\
\hline $\mathrm{N}(3)-\mathrm{C}(8)$ & $1.331(2)$ & $\mathrm{C}(31)-\mathrm{C}(32)$ & $1.532(3)$ \\
\hline $\mathrm{N}(3)-\mathrm{C}(34)$ & $1.450(3)$ & $C(34)-C(35)$ & $1.394(3)$ \\
\hline $\mathrm{N}(4)-\mathrm{C}(10)$ & $1.346(2)$ & $C(34)-C(39)$ & $1.399(3)$ \\
\hline$C(1)-C(2)$ & $1.493(3)$ & $C(35)-C(36)$ & $1.384(4)$ \\
\hline$C(2)-C(3)$ & $1.437(3)$ & $C(35)-C(40)$ & $1.514(5)$ \\
\hline$C(3)-C(4)$ & $1.382(3)$ & $C(36)-C(37)$ & $1.368(6)$ \\
\hline$C(4)-C(5)$ & $1.388(3)$ & $\mathrm{C}(37)-\mathrm{C}(38)$ & $1.356(6)$ \\
\hline$C(5)-C(6)$ & $1.378(3)$ & $\mathrm{C}(38)-\mathrm{C}(39)$ & $1.394(4)$ \\
\hline$C(6)-C(7)$ & $1.390(3)$ & $C(39)-C(43)$ & $1.500(4)$ \\
\hline $\mathrm{C}(7)-\mathrm{C}(8)$ & $1.431(3)$ & $\mathrm{C}(40)-\mathrm{C}(41)$ & $1.507(5)$ \\
\hline $\mathrm{C}(8)-\mathrm{C}(9)$ & $1.497(3)$ & $C(40)-C(42)$ & $1.520(5)$ \\
\hline$C(10)-C(15)$ & $1.430(3)$ & $C(43)-C(45)$ & $1.533(3)$ \\
\hline $\mathrm{C}(10)-\mathrm{C}(11)$ & $1.428(3)$ & $C(43)-C(44)$ & $1.563(4)$ \\
\hline $\mathrm{C}(11)-\mathrm{C}(12)$ & $1.381(3)$ & & \\
\hline$C(11)-C(16)$ & $1.516(3)$ & $\mathrm{N}(4)-\mathrm{Fe}(1)-\mathrm{N}(2)$ & $138.79(7)$ \\
\hline $\mathrm{C}(12)-\mathrm{C}(13)$ & $1.372(3)$ & $\mathrm{N}(4)-\mathrm{Fe}(1)-\mathrm{N}(3)$ & $105.44(7)$ \\
\hline$C(13)-C(14)$ & $1.380(3)$ & $\mathrm{N}(2)-\mathrm{Fe}(1)-\mathrm{N}(3)$ & 79.72(7) \\
\hline$C(14)-C(15)$ & $1.382(3)$ & $\mathrm{N}(4)-\mathrm{Fe}(1)-\mathrm{N}(1)$ & $104.65(7)$ \\
\hline$C(15)-C(19)$ & $1.512(3)$ & $\mathrm{N}(2)-\mathrm{Fe}(1)-\mathrm{N}(1)$ & $78.89(7)$ \\
\hline$C(16)-C(17)$ & $1.512(4)$ & $\mathrm{N}(3)-\mathrm{Fe}(1)-\mathrm{N}(1)$ & $149.86(6)$ \\
\hline$C(16)-C(18)$ & $1.535(4)$ & $\mathrm{C}(2)-\mathrm{N}(1)-\mathrm{C}(22)$ & $121.39(16)$ \\
\hline$C(19)-C(21)$ & $1.526(3)$ & $\mathrm{C}(2)-\mathrm{N}(1)-\mathrm{Fe}(1)$ & $114.31(12)$ \\
\hline C(19)-C(20) & $1.537(3)$ & $\mathrm{C}(22)-\mathrm{N}(1)-\mathrm{Fe}(1)$ & $123.99(11)$ \\
\hline$C(22)-C(23)$ & $1.401(3)$ & $\mathrm{C}(7)-\mathrm{N}(2)-\mathrm{C}(3)$ & $120.47(16)$ \\
\hline $\mathrm{C}(22)-\mathrm{C}(27)$ & $1.404(3)$ & $\mathrm{C}(7)-\mathrm{N}(2)-\mathrm{Fe}(1)$ & $119.37(13)$ \\
\hline$C(23)-C(24)$ & $1.397(3)$ & $\mathrm{C}(3)-\mathrm{N}(2)-\mathrm{Fe}(1)$ & $120.13(13)$ \\
\hline
\end{tabular}




\begin{tabular}{|c|c|c|c|}
\hline $\mathrm{C}(8)-\mathrm{N}(3)-\mathrm{C}(34)$ & $119.18(17)$ & $\mathrm{C}(21)-\mathrm{C}(19)-\mathrm{C}(20)$ & $109.4(2)$ \\
\hline $\mathrm{C}(8)-\mathrm{N}(3)-\mathrm{Fe}(1)$ & $114.80(13)$ & $\mathrm{C}(23)-\mathrm{C}(22)-\mathrm{C}(27)$ & $121.90(19)$ \\
\hline $\mathrm{C}(34)-\mathrm{N}(3)-\mathrm{Fe}(1)$ & $126.01(13)$ & $\mathrm{C}(23)-\mathrm{C}(22)-\mathrm{N}(1)$ & $117.96(19)$ \\
\hline $\mathrm{C}(10)-\mathrm{N}(4)-\mathrm{Fe}(1)$ & $165.68(15)$ & $\mathrm{C}(27)-\mathrm{C}(22)-\mathrm{N}(1)$ & $119.99(17)$ \\
\hline N(1)-C(2)-C(3) & $113.67(17)$ & $\mathrm{C}(24)-\mathrm{C}(23)-\mathrm{C}(22)$ & $117.1(2)$ \\
\hline $\mathrm{N}(1)-\mathrm{C}(2)-\mathrm{C}(1)$ & $125.59(18)$ & $\mathrm{C}(24)-\mathrm{C}(23)-\mathrm{C}(28)$ & $120.9(2)$ \\
\hline $\mathrm{C}(3)-\mathrm{C}(2)-\mathrm{C}(1)$ & $120.73(17)$ & $\mathrm{C}(22)-\mathrm{C}(23)-\mathrm{C}(28)$ & $122.00(19)$ \\
\hline $\mathrm{N}(2)-\mathrm{C}(3)-\mathrm{C}(4)$ & $119.89(19)$ & $C(25)-C(24)-C(23)$ & $121.7(2)$ \\
\hline $\mathrm{N}(2)-\mathrm{C}(3)-\mathrm{C}(2)$ & $111.23(16)$ & $\mathrm{C}(26)-\mathrm{C}(25)-\mathrm{C}(24)$ & $120.0(2)$ \\
\hline $\mathrm{C}(4)-\mathrm{C}(3)-\mathrm{C}(2)$ & $128.8(2)$ & $C(25)-C(26)-C(27)$ & $121.5(3)$ \\
\hline$C(3)-C(4)-C(5)$ & $119.6(2)$ & $\mathrm{C}(26)-\mathrm{C}(27)-\mathrm{C}(22)$ & $117.6(2)$ \\
\hline$C(6)-C(5)-C(4)$ & $120.2(2)$ & $\mathrm{C}(26)-\mathrm{C}(27)-\mathrm{C}(31)$ & $119.6(2)$ \\
\hline$C(5)-C(6)-C(7)$ & $119.7(2)$ & $\mathrm{C}(22)-\mathrm{C}(27)-\mathrm{C}(31)$ & $122.74(17)$ \\
\hline $\mathrm{N}(2)-\mathrm{C}(7)-\mathrm{C}(6)$ & 119.81(19) & $\mathrm{C}(29)-\mathrm{C}(28)-\mathrm{C}(30)$ & $108.2(3)$ \\
\hline N(2)-C(7)-C(8) & $111.09(17)$ & $\mathrm{C}(29)-\mathrm{C}(28)-\mathrm{C}(23)$ & $112.0(2)$ \\
\hline$C(6)-C(7)-C(8)$ & 129.1(2) & $\mathrm{C}(30)-\mathrm{C}(28)-\mathrm{C}(23)$ & $113.1(3)$ \\
\hline N(3)-C(8)-C(7) & $113.45(18)$ & $C(27)-C(31)-C(33)$ & $113.0(2)$ \\
\hline $\mathrm{N}(3)-\mathrm{C}(8)-\mathrm{C}(9)$ & $125.6(2)$ & $\mathrm{C}(27)-\mathrm{C}(31)-\mathrm{C}(32)$ & $110.84(18)$ \\
\hline $\mathrm{C}(7)-\mathrm{C}(8)-\mathrm{C}(9)$ & $120.87(19)$ & $\mathrm{C}(33)-\mathrm{C}(31)-\mathrm{C}(32)$ & $109.38(19)$ \\
\hline N(4)-C(10)-C(15) & $120.38(17)$ & $\mathrm{C}(35)-\mathrm{C}(34)-\mathrm{C}(39)$ & $121.0(2)$ \\
\hline N(4)-C(10)-C(11) & $120.22(18)$ & $\mathrm{C}(35)-\mathrm{C}(34)-\mathrm{N}(3)$ & $116.8(2)$ \\
\hline$C(15)-C(10)-C(11)$ & $119.39(17)$ & $\mathrm{C}(39)-\mathrm{C}(34)-\mathrm{N}(3)$ & $122.15(19)$ \\
\hline $\mathrm{C}(12)-\mathrm{C}(11)-\mathrm{C}(10)$ & $118.6(2)$ & $\mathrm{C}(36)-\mathrm{C}(35)-\mathrm{C}(34)$ & $118.0(3)$ \\
\hline$C(12)-C(11)-C(16)$ & $122.7(2)$ & $\mathrm{C}(36)-\mathrm{C}(35)-\mathrm{C}(40)$ & 119.1(3) \\
\hline$C(10)-C(11)-C(16)$ & 118.71(18) & $\mathrm{C}(34)-\mathrm{C}(35)-\mathrm{C}(40)$ & $122.9(2)$ \\
\hline $\mathrm{C}(13)-\mathrm{C}(12)-\mathrm{C}(11)$ & $121.9(2)$ & $\mathrm{C}(37)-\mathrm{C}(36)-\mathrm{C}(35)$ & $121.8(3)$ \\
\hline$C(12)-C(13)-C(14)$ & $119.9(2)$ & $\mathrm{C}(38)-\mathrm{C}(37)-\mathrm{C}(36)$ & $119.7(3)$ \\
\hline$C(13)-C(14)-C(15)$ & $121.8(2)$ & $\mathrm{C}(37)-\mathrm{C}(38)-\mathrm{C}(39)$ & $121.6(3)$ \\
\hline$C(14)-C(15)-C(10)$ & $118.45(19)$ & $\mathrm{C}(38)-\mathrm{C}(39)-\mathrm{C}(34)$ & $117.9(3)$ \\
\hline $\mathrm{C}(14)-\mathrm{C}(15)-\mathrm{C}(19)$ & $122.2(2)$ & $\mathrm{C}(38)-\mathrm{C}(39)-\mathrm{C}(43)$ & $117.4(3)$ \\
\hline$C(10)-C(15)-C(19)$ & $119.37(18)$ & $\mathrm{C}(34)-\mathrm{C}(39)-\mathrm{C}(43)$ & $124.8(2)$ \\
\hline $\mathrm{C}(17)-\mathrm{C}(16)-\mathrm{C}(11)$ & $114.4(2)$ & $\mathrm{C}(41)-\mathrm{C}(40)-\mathrm{C}(35)$ & 111.1(3) \\
\hline$C(17)-C(16)-C(18)$ & $111.0(2)$ & $C(41)-C(40)-C(42)$ & $109.7(3)$ \\
\hline $\mathrm{C}(11)-\mathrm{C}(16)-\mathrm{C}(18)$ & $110.1(2)$ & $C(35)-C(40)-C(42)$ & $111.8(3)$ \\
\hline $\mathrm{C}(15)-\mathrm{C}(19)-\mathrm{C}(21)$ & $112.44(19)$ & $\mathrm{C}(39)-\mathrm{C}(43)-\mathrm{C}(45)$ & $111.6(2)$ \\
\hline$C(15)-C(19)-C(20)$ & $113.4(2)$ & $\mathrm{C}(39)-\mathrm{C}(43)-\mathrm{C}(44)$ & $111.6(2)$ \\
\hline
\end{tabular}




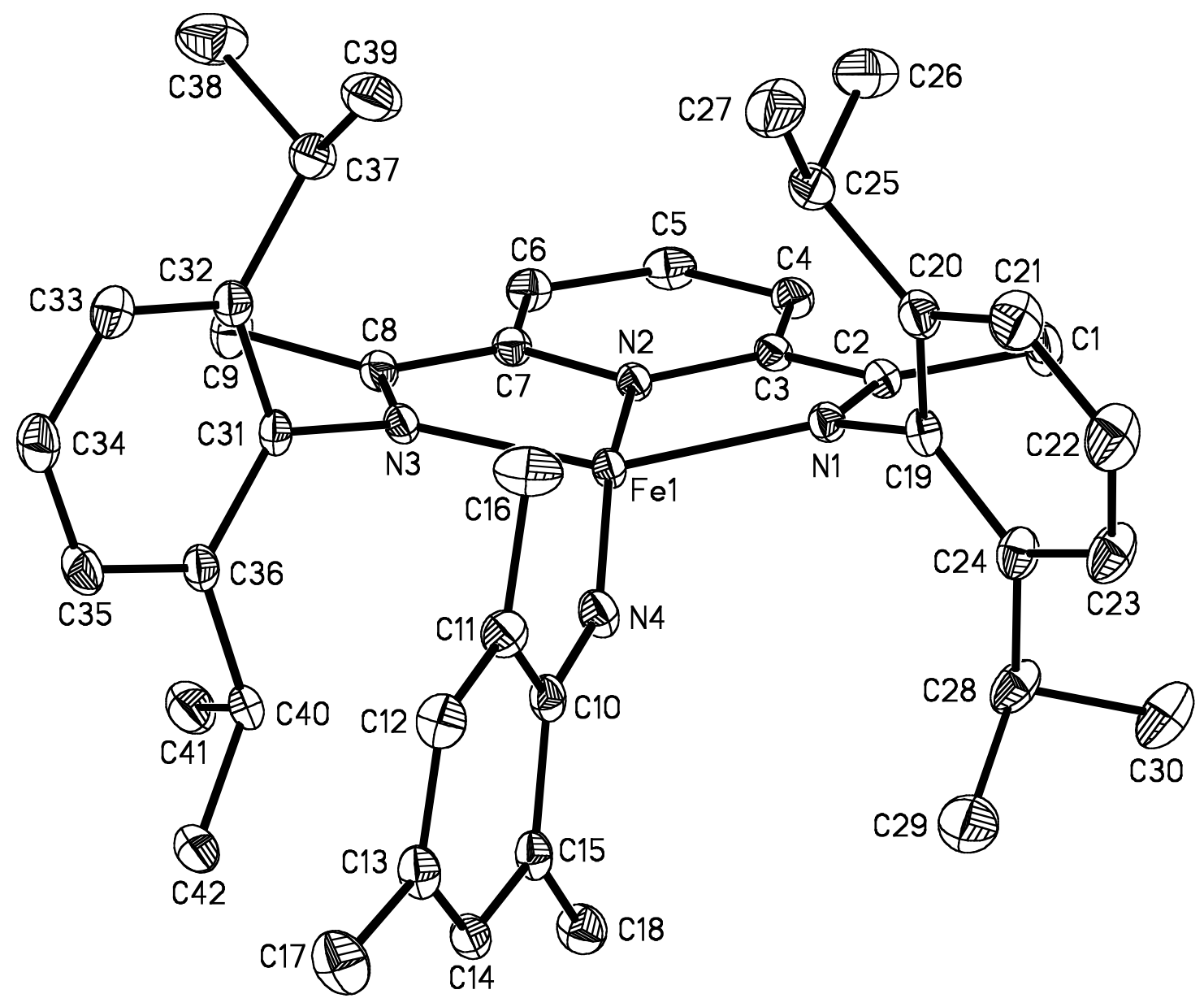

Figure S2. Fully labeled view of the molecular structure of $\mathbf{2 d}$ at $30 \%$ probability ellipsoids. Hydrogen atoms omitted for clarity. 
TableS3. Crystal data and structure refinement for $\mathbf{2 d}$.

Identification code

Empirical formula

Formula weight

Temperature

Wavelength

Crystal system

Space group

Unit cell dimensions

Volume

Z

Density (calculated)

Absorption coefficient

$\mathrm{F}(000)$

Crystal size

Theta range for data collection

Index ranges

Reflections collected

Independent reflections

Completeness to theta $=26.84^{\circ}$

Absorption correction

Max. and min. transmission

Refinement method

Data / restraints / parameters

Goodness-of-fit on $\mathrm{F}^{2}$

Final $\mathrm{R}$ indices $[\mathrm{I}>2 \operatorname{sigma}(\mathrm{I})]$

$\mathrm{R}$ indices (all data)

Largest diff. peak and hole scb46

C42 H54 Fe N4

670.74

173(2) K

$0.71073 \AA$

Triclinic

P-1

$$
\begin{array}{ll}
\mathrm{a}=10.5181(8) \AA & \alpha=96.056(4)^{\circ} . \\
\mathrm{b}=12.1422(11) \AA & \beta=93.086(4)^{\circ} . \\
\mathrm{c}=15.0667(14) \AA & \gamma=103.537(3)^{\circ} .
\end{array}
$$

1854.2(3) $\AA^{3}$

2

$1.201 \mathrm{Mg} / \mathrm{m}^{3}$

$0.441 \mathrm{~mm}^{-1}$

720

$0.50 \times 0.40 \times 0.03 \mathrm{~mm}^{3}$

1.74 to $26.84^{\circ}$.

$-13<=\mathrm{h}<=13,-15<=\mathrm{k}<=15,-19<=\mathrm{l}<=19$

31438

$7753[\mathrm{R}($ int $)=0.0378]$

$97.4 \%$

Semi-empirical from equivalents

0.9869 and 0.8098

Full-matrix least-squares on $\mathrm{F}^{2}$

7753 / 0 / 607

1.029

$\mathrm{R} 1=0.0365, \mathrm{wR} 2=0.0875$

$\mathrm{R} 1=0.0558, \mathrm{wR} 2=0.0952$

0.321 and -0.321 e. $\AA^{-3}$ 
Table S4. Bond lengths $[\AA]$ and angles $\left[{ }^{\circ}\right]$ for $\mathbf{2 d}$.

\begin{tabular}{|c|c|c|c|}
\hline $\mathrm{Fe}(1)-\mathrm{N}(4)$ & $1.7165(15)$ & $C(23)-C(24)$ & $1.388(3)$ \\
\hline $\mathrm{Fe}(1)-\mathrm{N}(2)$ & $1.8718(14)$ & $C(24)-C(28)$ & $1.515(3)$ \\
\hline $\mathrm{Fe}(1)-\mathrm{N}(3)$ & $1.9786(14)$ & $C(25)-C(26)$ & $1.525(3)$ \\
\hline $\mathrm{Fe}(1)-\mathrm{N}(1)$ & $2.0105(14)$ & $C(25)-C(27)$ & $1.526(3)$ \\
\hline $\mathrm{N}(1)-\mathrm{C}(2)$ & $1.321(2)$ & $C(28)-C(29)$ & $1.522(4)$ \\
\hline $\mathrm{N}(1)-\mathrm{C}(19)$ & $1.441(2)$ & $C(28)-C(30)$ & $1.531(3)$ \\
\hline $\mathrm{N}(2)-\mathrm{C}(3)$ & $1.373(2)$ & $C(31)-C(32)$ & $1.403(2)$ \\
\hline $\mathrm{N}(2)-\mathrm{C}(7)$ & $1.374(2)$ & $C(31)-C(36)$ & $1.405(3)$ \\
\hline $\mathrm{N}(3)-\mathrm{C}(8)$ & $1.334(2)$ & $C(32)-C(33)$ & $1.392(3)$ \\
\hline $\mathrm{N}(3)-\mathrm{C}(31)$ & $1.446(2)$ & $\mathrm{C}(32)-\mathrm{C}(37)$ & $1.521(3)$ \\
\hline $\mathrm{N}(4)-\mathrm{C}(10)$ & $1.348(2)$ & $C(33)-C(34)$ & $1.373(3)$ \\
\hline$C(1)-C(2)$ & $1.497(2)$ & $C(34)-C(35)$ & $1.380(3)$ \\
\hline $\mathrm{C}(2)-\mathrm{C}(3)$ & $1.436(2)$ & $C(35)-C(36)$ & $1.393(3)$ \\
\hline $\mathrm{C}(3)-\mathrm{C}(4)$ & $1.386(2)$ & $C(36)-C(40)$ & $1.522(3)$ \\
\hline$C(4)-C(5)$ & $1.388(3)$ & $\mathrm{C}(37)-\mathrm{C}(39)$ & $1.517(3)$ \\
\hline$C(5)-C(6)$ & $1.384(3)$ & $\mathrm{C}(37)-\mathrm{C}(38)$ & $1.533(3)$ \\
\hline$C(6)-C(7)$ & $1.386(2)$ & $C(40)-C(42)$ & $1.520(3)$ \\
\hline $\mathrm{C}(7)-\mathrm{C}(8)$ & $1.426(2)$ & $\mathrm{C}(40)-\mathrm{C}(41)$ & $1.525(3)$ \\
\hline$C(8)-C(9)$ & $1.495(3)$ & & \\
\hline $\mathrm{C}(10)-\mathrm{C}(11)$ & $1.426(3)$ & $\mathrm{N}(4)-\mathrm{Fe}(1)-\mathrm{N}(2)$ & $154.75(7)$ \\
\hline$C(10)-C(15)$ & $1.431(3)$ & $\mathrm{N}(4)-\mathrm{Fe}(1)-\mathrm{N}(3)$ & $105.05(7)$ \\
\hline $\mathrm{C}(11)-\mathrm{C}(12)$ & $1.376(3)$ & $\mathrm{N}(2)-\mathrm{Fe}(1)-\mathrm{N}(3)$ & $78.99(6)$ \\
\hline $\mathrm{C}(11)-\mathrm{C}(16)$ & $1.497(3)$ & $\mathrm{N}(4)-\mathrm{Fe}(1)-\mathrm{N}(1)$ & $102.18(6)$ \\
\hline $\mathrm{C}(12)-\mathrm{C}(13)$ & $1.388(3)$ & $\mathrm{N}(2)-\mathrm{Fe}(1)-\mathrm{N}(1)$ & $78.42(6)$ \\
\hline$C(13)-C(14)$ & $1.390(3)$ & $\mathrm{N}(3)-\mathrm{Fe}(1)-\mathrm{N}(1)$ & $152.37(6)$ \\
\hline$C(13)-C(17)$ & $1.500(3)$ & $\mathrm{C}(2)-\mathrm{N}(1)-\mathrm{C}(19)$ & $118.78(14)$ \\
\hline$C(14)-C(15)$ & $1.382(3)$ & $\mathrm{C}(2)-\mathrm{N}(1)-\mathrm{Fe}(1)$ & $115.53(11)$ \\
\hline$C(15)-C(18)$ & $1.499(3)$ & $\mathrm{C}(19)-\mathrm{N}(1)-\mathrm{Fe}(1)$ & $125.69(11)$ \\
\hline C(19)-C(24) & $1.395(3)$ & $\mathrm{C}(3)-\mathrm{N}(2)-\mathrm{C}(7)$ & $119.84(14)$ \\
\hline C(19)-C(20) & $1.413(3)$ & $\mathrm{C}(3)-\mathrm{N}(2)-\mathrm{Fe}(1)$ & $120.33(11)$ \\
\hline$C(20)-C(21)$ & $1.384(3)$ & $\mathrm{C}(7)-\mathrm{N}(2)-\mathrm{Fe}(1)$ & $119.83(11)$ \\
\hline$C(20)-C(25)$ & $1.521(3)$ & $\mathrm{C}(8)-\mathrm{N}(3)-\mathrm{C}(31)$ & $117.90(14)$ \\
\hline$C(21)-C(22)$ & $1.382(3)$ & $\mathrm{C}(8)-\mathrm{N}(3)-\mathrm{Fe}(1)$ & $116.23(11)$ \\
\hline$C(22)-C(23)$ & $1.374(3)$ & $\mathrm{C}(31)-\mathrm{N}(3)-\mathrm{Fe}(1)$ & $125.70(11)$ \\
\hline
\end{tabular}




\begin{tabular}{|c|c|c|c|}
\hline $\mathrm{C}(10)-\mathrm{N}(4)-\mathrm{Fe}(1)$ & $159.00(13)$ & $C(19)-C(20)-C(25)$ & $120.89(17)$ \\
\hline $\mathrm{N}(1)-\mathrm{C}(2)-\mathrm{C}(3)$ & $114.04(15)$ & $\mathrm{C}(22)-\mathrm{C}(21)-\mathrm{C}(20)$ & $121.5(2)$ \\
\hline $\mathrm{N}(1)-\mathrm{C}(2)-\mathrm{C}(1)$ & $124.71(16)$ & $C(23)-C(22)-C(21)$ & $119.8(2)$ \\
\hline $\mathrm{C}(3)-\mathrm{C}(2)-\mathrm{C}(1)$ & $121.14(16)$ & $\mathrm{C}(22)-\mathrm{C}(23)-\mathrm{C}(24)$ & $121.3(2)$ \\
\hline $\mathrm{N}(2)-\mathrm{C}(3)-\mathrm{C}(4)$ & $120.49(16)$ & $C(23)-C(24)-C(19)$ & $118.17(17)$ \\
\hline $\mathrm{N}(2)-\mathrm{C}(3)-\mathrm{C}(2)$ & $110.53(15)$ & $\mathrm{C}(23)-\mathrm{C}(24)-\mathrm{C}(28)$ & $119.32(17)$ \\
\hline $\mathrm{C}(4)-\mathrm{C}(3)-\mathrm{C}(2)$ & $128.90(16)$ & $C(19)-C(24)-C(28)$ & $122.44(16)$ \\
\hline$C(3)-C(4)-C(5)$ & $119.63(17)$ & $\mathrm{C}(20)-\mathrm{C}(25)-\mathrm{C}(26)$ & $112.67(17)$ \\
\hline C(6)-C(5)-C(4) & $119.66(18)$ & $C(20)-C(25)-C(27)$ & $112.9(2)$ \\
\hline$C(5)-C(6)-C(7)$ & $119.89(17)$ & $C(26)-C(25)-C(27)$ & $109.5(2)$ \\
\hline $\mathrm{N}(2)-\mathrm{C}(7)-\mathrm{C}(6)$ & $120.32(16)$ & $\mathrm{C}(24)-\mathrm{C}(28)-\mathrm{C}(29)$ & $109.96(18)$ \\
\hline $\mathrm{N}(2)-\mathrm{C}(7)-\mathrm{C}(8)$ & $110.92(15)$ & $\mathrm{C}(24)-\mathrm{C}(28)-\mathrm{C}(30)$ & $112.65(19)$ \\
\hline $\mathrm{C}(6)-\mathrm{C}(7)-\mathrm{C}(8)$ & $128.71(16)$ & $\mathrm{C}(29)-\mathrm{C}(28)-\mathrm{C}(30)$ & $109.4(2)$ \\
\hline $\mathrm{N}(3)-\mathrm{C}(8)-\mathrm{C}(7)$ & $113.56(15)$ & $\mathrm{C}(32)-\mathrm{C}(31)-\mathrm{C}(36)$ & $121.92(16)$ \\
\hline $\mathrm{N}(3)-\mathrm{C}(8)-\mathrm{C}(9)$ & $125.31(16)$ & $\mathrm{C}(32)-\mathrm{C}(31)-\mathrm{N}(3)$ & $117.69(15)$ \\
\hline $\mathrm{C}(7)-\mathrm{C}(8)-\mathrm{C}(9)$ & $121.03(17)$ & $\mathrm{C}(36)-\mathrm{C}(31)-\mathrm{N}(3)$ & $120.38(15)$ \\
\hline N(4)-C(10)-C(11) & $121.11(17)$ & $\mathrm{C}(33)-\mathrm{C}(32)-\mathrm{C}(31)$ & $117.87(17)$ \\
\hline N(4)-C(10)-C(15) & $121.12(16)$ & $\mathrm{C}(33)-\mathrm{C}(32)-\mathrm{C}(37)$ & $119.22(17)$ \\
\hline$C(11)-C(10)-C(15)$ & $117.77(16)$ & $\mathrm{C}(31)-\mathrm{C}(32)-\mathrm{C}(37)$ & $122.91(16)$ \\
\hline$C(12)-C(11)-C(10)$ & $119.67(18)$ & $C(34)-C(33)-C(32)$ & $121.23(19)$ \\
\hline$C(12)-C(11)-C(16)$ & $121.70(19)$ & $\mathrm{C}(33)-\mathrm{C}(34)-\mathrm{C}(35)$ & $120.13(19)$ \\
\hline$C(10)-C(11)-C(16)$ & 118.63(18) & $C(34)-C(35)-C(36)$ & $121.47(19)$ \\
\hline$C(11)-C(12)-C(13)$ & $122.86(18)$ & $\mathrm{C}(35)-\mathrm{C}(36)-\mathrm{C}(31)$ & $117.37(17)$ \\
\hline$C(12)-C(13)-C(14)$ & $117.35(18)$ & $\mathrm{C}(35)-\mathrm{C}(36)-\mathrm{C}(40)$ & $120.78(17)$ \\
\hline $\mathrm{C}(12)-\mathrm{C}(13)-\mathrm{C}(17)$ & $121.5(2)$ & $C(31)-C(36)-C(40)$ & $121.85(16)$ \\
\hline$C(14)-C(13)-C(17)$ & 121.1(2) & $\mathrm{C}(39)-\mathrm{C}(37)-\mathrm{C}(32)$ & $112.19(18)$ \\
\hline$C(15)-C(14)-C(13)$ & $122.73(18)$ & $\mathrm{C}(39)-\mathrm{C}(37)-\mathrm{C}(38)$ & $108.71(18)$ \\
\hline$C(14)-C(15)-C(10)$ & $119.37(17)$ & $\mathrm{C}(32)-\mathrm{C}(37)-\mathrm{C}(38)$ & $111.76(18)$ \\
\hline$C(14)-C(15)-C(18)$ & $120.61(18)$ & $C(42)-C(40)-C(36)$ & $113.07(18)$ \\
\hline $\mathrm{C}(10)-\mathrm{C}(15)-\mathrm{C}(18)$ & $119.96(17)$ & $\mathrm{C}(42)-\mathrm{C}(40)-\mathrm{C}(41)$ & $109.48(18)$ \\
\hline$C(24)-C(19)-C(20)$ & $121.53(16)$ & $C(36)-C(40)-C(41)$ & $112.12(17)$ \\
\hline C(24)-C(19)-N(1) & $121.39(15)$ & & \\
\hline $\mathrm{C}(20)-\mathrm{C}(19)-\mathrm{N}(1)$ & $117.08(15)$ & & \\
\hline$C(21)-C(20)-C(19)$ & $117.58(18)$ & & \\
\hline $\mathrm{C}(21)-\mathrm{C}(20)-\mathrm{C}(25)$ & $121.53(18)$ & & \\
\hline
\end{tabular}




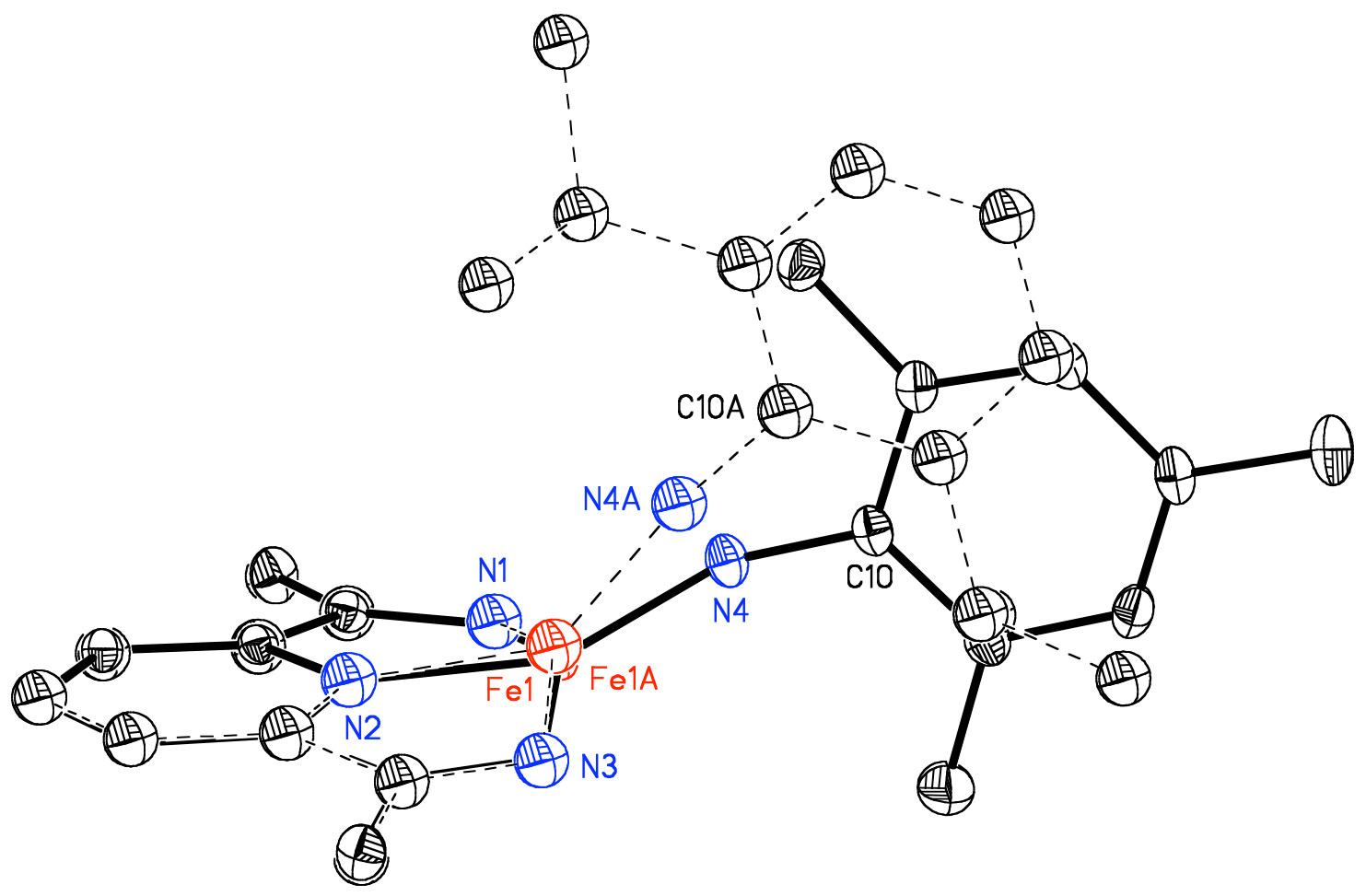

Figure S3. Comparison of the core of the solid state structures of $\mathbf{2 a}$ and $\mathbf{2 d}$. 

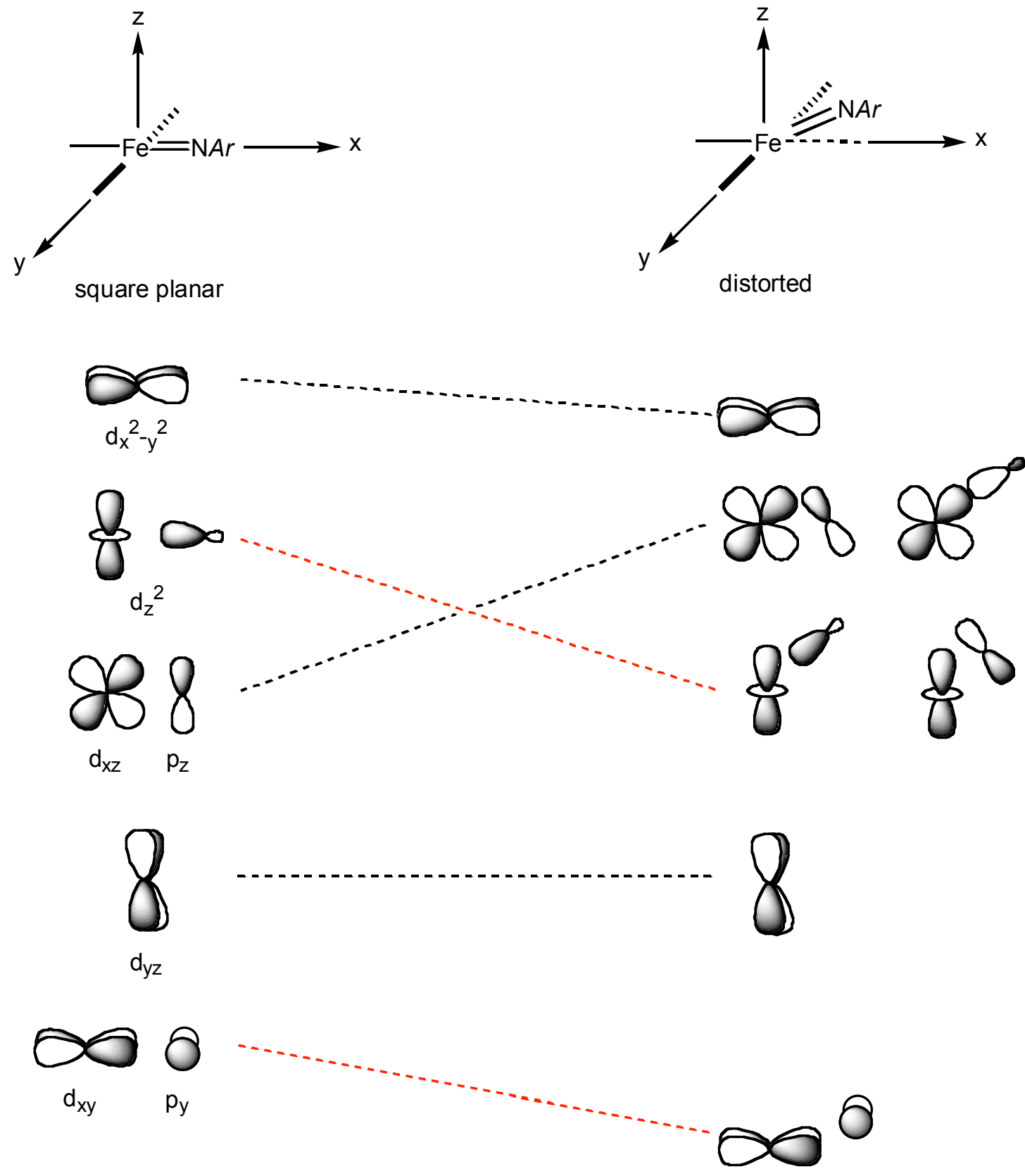

Figure S4. Qualitative correlation diagram for the distortion of iron imide compounds from planarity. Adapted from Albright, Wangbo, Burdett Orbital Interactions in Chemistry, Wiley, New York, 1985. 


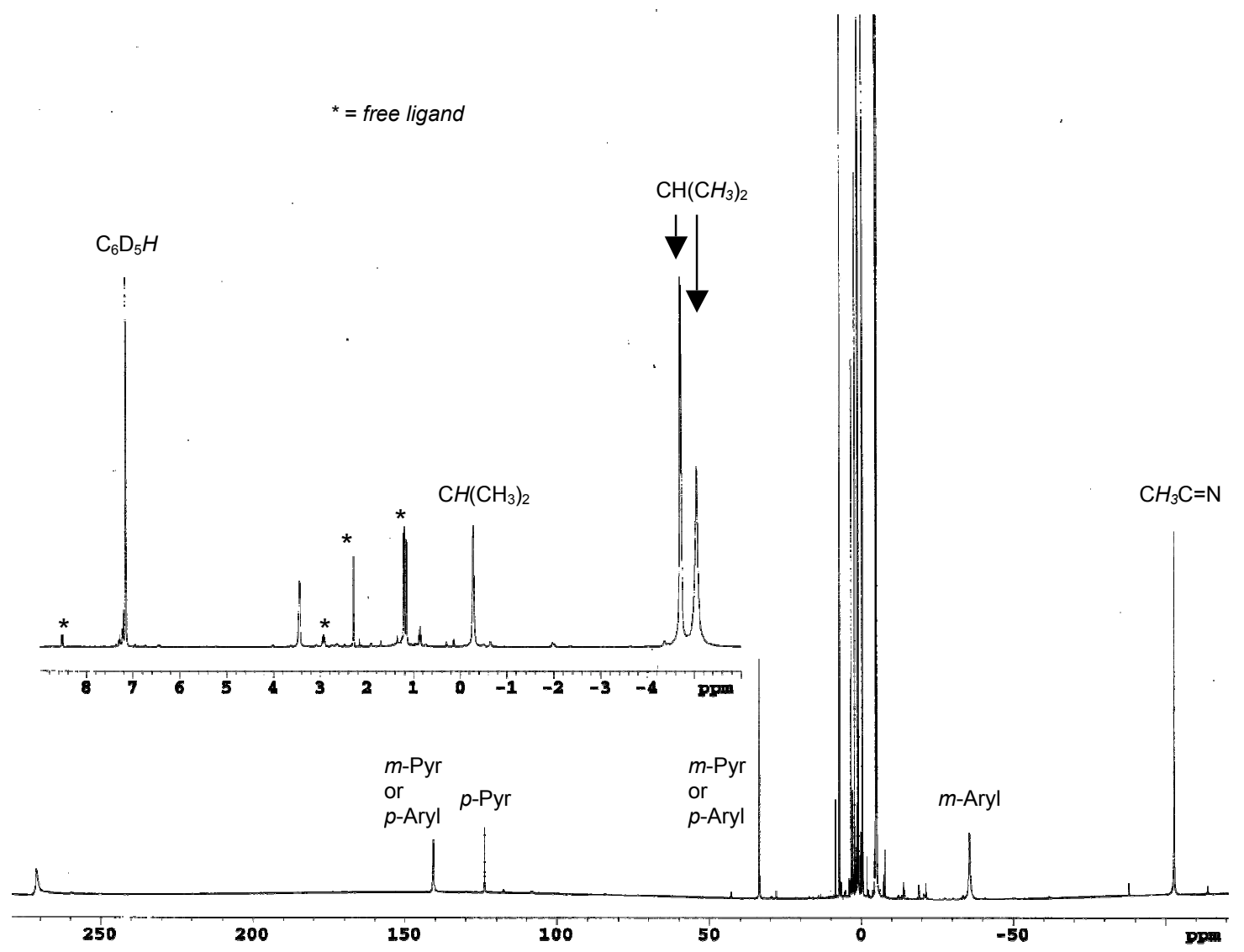

Figure S5. ${ }^{1} \mathrm{H}$ NMR spectrum of $\mathbf{2 d}$ in benzene- $d_{6}$ at $23{ }^{\circ} \mathrm{C}$. 
CV of PDIFe(NDIPP)

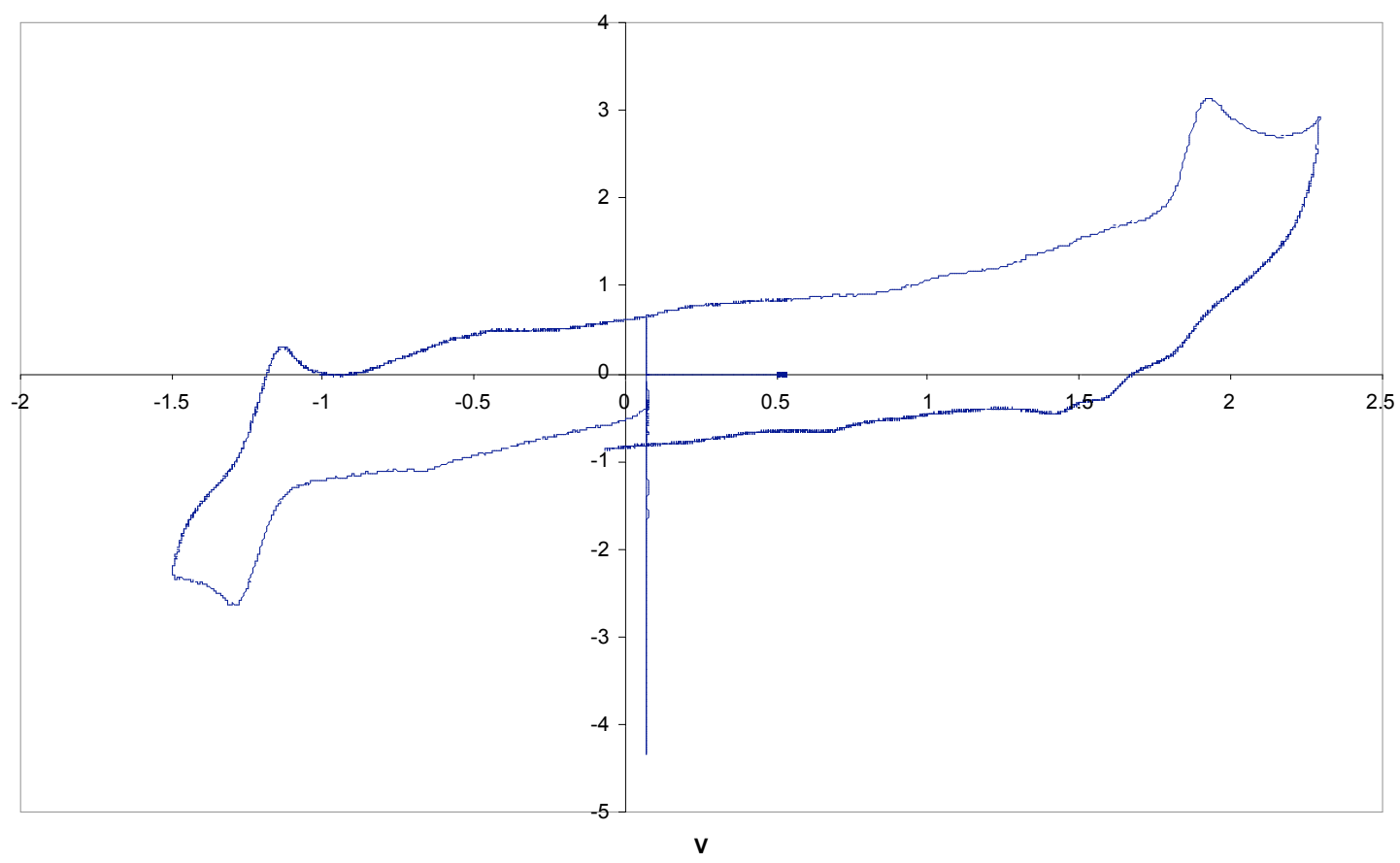

Figure S6. Cyclic voltamogram of $\mathbf{2 a}$ in THF with $\left[n-\mathrm{Bu}_{4} \mathrm{~N}\right]\left[\mathrm{PF}_{6}\right]$ electrolyte and a scan rate of 100 $\mathrm{mV} / \mathrm{sec}$. 


\section{References}

${ }^{1}$ Pangborn, A.B.; Giardello, M.A.; Grubbs, R.H.; Rosen, R.K.; Timmers, F.J. Organometallics 1996, 15, 1518.

${ }^{2}$ Lui, Q.; Tor, Y. Org. Lett. 2003, 5, 2571.

${ }^{3}$ Bart, S. C.; Lobkovsky, E.; Chirik, P. J. J. Am. Chem. Soc. 2004, 126, 13794. 